\title{
Fine-Tuning Transformers for Identifying Self-Reporting Potential Cases and Symptoms of COVID-19 in Tweets
}

\author{
Max Fleming ${ }^{1}$ Priyanka Dondeti $^{2}$ Caitlin N. Dreisbach $^{3}$ Adam Poliak $^{2,3}$ \\ Johns Hopkins University ${ }^{1}$ Barnard College ${ }^{2}$ \\ Data Science Institute, Columbia University ${ }^{3}$ \\ mflemi21@jhu.edu, \{pdd2112, apoliak\}@barnard.edu, c.dreisbach@columbia.edu
}

\begin{abstract}
We describe our straight-forward approach for Tasks 5 and 6 of 2021 Social Media Mining for Health Applications (SMM4H) shared tasks. Our system is based on fine-tuning DistillBERT on each task, as well as first fine-tuning the model on the other task. We explore how much fine-tuning is necessary for accurately classifying tweets as containing self-reported COVID-19 symptoms (Task 5) or whether a tweet related to COVID-19 is self-reporting, non-personal reporting, or a literature/news mention of the virus (Task 6).
\end{abstract}

\section{Introduction}

Fine-tuning off-the-shelf Transformer-based contextualized language models is a common baseline for contemporary Natural Language Processing (Ruder, 2021). When developing our system for Task 6 of the 2021 Social Media Mining for Health Applications (SMM4H), we quickly discovered that fine-tuning DistilBERT (Sanh et al., 2019), a smaller and distilled version of BERT (Devlin et al., 2019), outperformed training traditional, non-neural machine learning models. Fine-tuning DistilBERT on the released training set resulted in a micro-F1 of 97.60 on the Task 6 release development set. While this approach was not as successful for Task 5 (binary-F1 of 51.49), in this paper, we explore how much fine-tuning is necessary for these tasks and whether there are benefits to first training the model on the other task since both are related to COVID-19. ${ }^{1}$

\section{Task Description}

Both Task 5 and Task 6 focused on classifying tweets related to COVID-19 (Magge et al., 2021). Task 5 required classifying tweets as describing self-reporting potential cases of COVID-19 or not.

\footnotetext{
${ }^{1}$ All code developed is publicly available at https : / / github.com/mfleming99/SMM4H_2021.
}

Tweets were extracted via manually crafted regular expressions for potential self-reported mentions of COVID-19 and then annotated by two people. 1,148 Tweets were labeled as containing a selfreporting potential cases and 6,033 were labeled as "Other." The other tweets that might discuss COVID-19 but do not specifically reporting a user's or their household's potential cases were labeled as "Other." ${ }^{2}$ Systems were ranked by F1-score for the "potential case" class.

In Task 6, systems must determine whether a tweet related to COVID-19 is self-reporting, nonpersonal reporting, or a literature/news mention of the virus. 1, 421 released examples are labeled as self-reporting, 3, 567 as non-personal reports, and 4,464 as literature/news mentions. Systems were evaluated by micro-F1 score. Table 1 includes examples tweets from the development sets.

\section{Method}

We fine-tuned DistillBERT using the implementation developed and released by HuggingFace transformer's library (Wolf et al., 2020). We trained the model for 3 epochs, using a batch size of 64 examples, warm-up steps of 500 for the learning rate scheduler and a weight decay of 0.01 . Following Peters et al. (2019) recommendation to add minimal task hyper-parameters when finetuning pre-trained models, we used the remaining default hyper-parameters from the library's Trainer class. All models were trained across 2 NVIDIA RTX 3090's.

\subsection{Cross-validation}

We used 5-fold evaluation to determine the utility of this simple approach. For each task, we combined the training and development sets and removed duplicate tweets, resulting in 7,174 and 9, 452 annotated examples for Task 5 and Task 6

\footnotetext{
${ }^{2}$ See Klein et al. (2021) for a detailed description of the data collection and annotation protocols.
} 


\begin{tabular}{c|l|c}
\hline Task & \multicolumn{1}{|c}{ Tweet } & Label \\
\hline Task5 & $\begin{array}{l}\text { Just in case I do manage to contract \#coronavirus during the social distancing } \\
\text { phase. I will kill it from the INSIDE! } \\
\text { So I've had this sore throat for a couple of days, I don't know if im being } \\
\text { dramatic but i'm scared its Coronavirus?? }\end{array}$ & Other \\
\hline Task6 & $\begin{array}{l}\text { New evidence suggests that neurological symptoms among hospitalized COVID- } \\
19 \text { patients are extremely common } \\
\text { My dad tested positive for COVID-19 earlier this week, started having difficulty } \\
\text { breathing this morning, and is now in the ED. } \\
\text { Covid week 13 update. Week 11 kidney pain on the wane, presenting as high } \\
\text { BP (affecting brain speed, vision, tightness in veins). }\end{array}$ & Lit-News \\
\hline
\end{tabular}

Table 1: Examples of tweets and labels for each task, abridged for space.
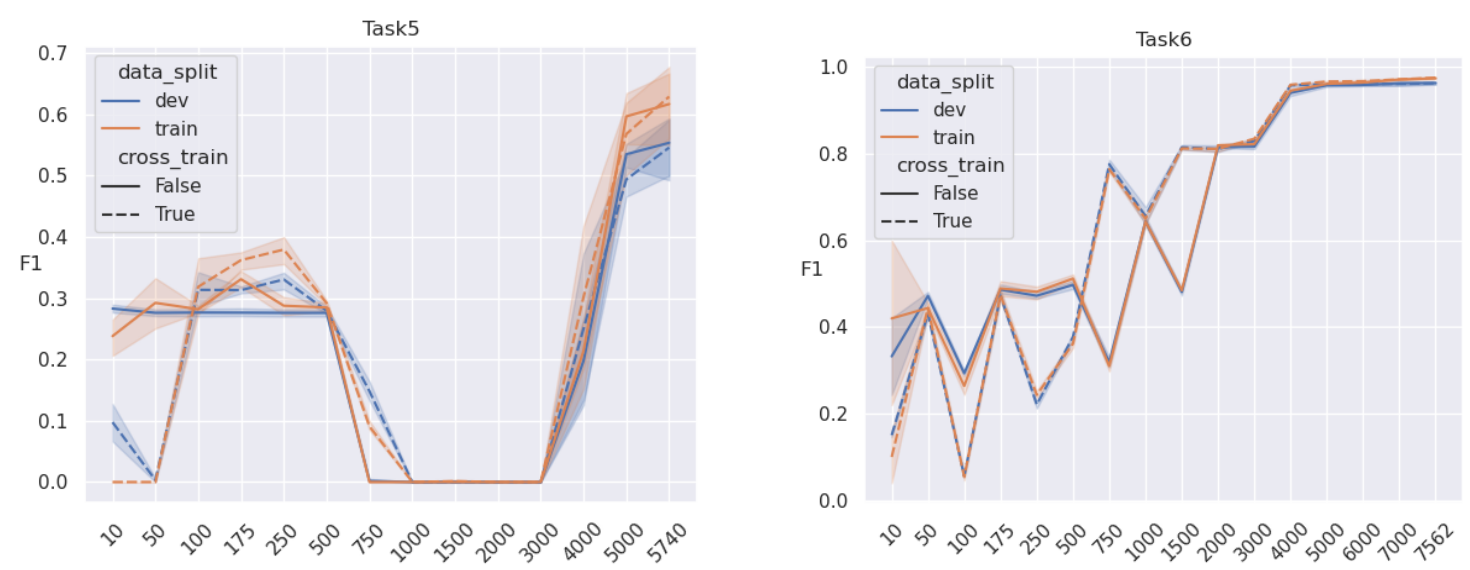

Figure 1: 5-fold results. The left and right graph respectively reflect binary-F1 results for Task 5 and micro-F1 results for Task 6. y-axes indicate F1 and x-axes indicate the number of training examples used. Dotted and solid lines, respectively, indicated that the model was pre-trained on the other task or not. Blue and orange respectively correspond to the training and development folds. The lines indicate the average across the 5 folds and the shaded areas indicate the range of results.

respectively. ${ }^{3}$ We divided the datasets into 5 folds of roughly 1,435 and 1,890 labeled examples for Task 5 and Task 6 and fine-tune models on 4 of the folds and test on the held out fold. For each fold, we fine-tuned the model on a increasing number of training examples: $10,50,100,175,250,500$, $750,1 \mathrm{~K}, 1.5 \mathrm{~K}, 2 \mathrm{~K}, 3 \mathrm{~K}, 4 \mathrm{~K}, 5 \mathrm{~K}, 6 \mathrm{~K}, 7 \mathrm{~K}, 8 \mathrm{~K} .{ }^{4} \mathrm{Ad}-$ ditionally, for both tasks, we experimented with using a model pre-trained on the other task. We hypothesized this might be beneficial as these tasks seem to be related.

\footnotetext{
${ }^{3} 7$ and 115 examples were removed for Task 5 and 6 respectively.

${ }^{4}$ For Task 5, the maximum number of training examples are 5,740
}

\section{Results}

Figure 1 shows the results of fine-tuning DistillBert on each task. For Task 5 (left graph), when finetuning on 50 examples or less, initially training on Task 6 (dotted lines) is detrimental. When finetuning on somewhere between 50 and 100 training examples, first training the models on Task 6 leads to a noticeable improvement. This continued until we fine-tuned the model on 500 examples. Once we fine-tuned the model on 1000 to 3000 examples, there is no difference between first training on the other task as the models only predict the majority class "Other". As the number of training examples increases from this point, we begin to see large improvements and larger variances between the models trained on different folds. First training on Task 6 appears to be most beneficial when fine- 
tuning on 100 through 750 Task 5 examples.

For Task 6 (right graph), the benefits of pretraining the model on Task 5 are not as clear cut, and the results oscillate a bit more. It seems that pre-training on Task 5 is only beneficial when finetuning the model on 750 through 2,000 examples (except for the case when fine-tuning on 1,000 examples). For both tasks, pre-training on the other task seems to make no difference once the model is fine-tuned on enough task specific examples (roughly 1,000 and 2,000 examples for Task 5 and Task 6).

Held out test set In these experiments, the model performance on the held out fold seems to increase as we add more training examples. While results for Task 6 seem to plateau, we notice a small increase as we continue to add training examples. Therefore, for our official submissions, we finetuned the model on all released examples.

Table 2 reports results for the official test sets. ${ }^{5}$ The 63.19 binary-F1 for Task 5 might indicate that training on more examples is beneficial for this task. For Task 6, we notice the micro-F1 drops a bit compared to the results on the held out folds. For both tasks, pre-training on the other task is not beneficial on the test set when trained on as many labeled examples as possible.

We also include a majority vote ensemble of the 5 -fold models trained on different training sizes. These test results follow the general trends in Figure 1 indicating when it is most beneficial to first train the DistilBert model on the other task. Similar to the results in Figure 1, when fine-tuning on 750 through 3,000 Task 5 examples, the model achieved a 0 binary-F1 since it always predicted the majority class "Other."

\section{Conclusion}

We discussed our straightforward approach of finetuning a DistilBert model on Tasks 5 and 6 of the 2021 Social Media Mining for Health Applications shared tasks. While not attaining state-of-the-art, these results are competitive and demonstrate the benefit of leveraging large scale pre-trained contextualized language models. We additionally explored the benefits of first training the model on the corresponding task and determine when this can be beneficial. Future work might consider jointly

\footnotetext{
${ }^{5}$ These numbers differ from the official leaderboard during the evaluation as we discovered a bug related to loading our pre-trained models during the post-evaluation period.
}

\begin{tabular}{c|cc|cc}
\hline Train Size & \multicolumn{2}{|c}{ Task5 } & \multicolumn{2}{c}{ Task6 } \\
& & & a & \\
\hline- & 63.19 & 62.24 & 92.88 & 91.77 \\
\hline 50 & 29.33 & 05.72 & 46.17 & 42.75 \\
100 & - & - & 27.65 & 05.72 \\
175 & 28.54 & 32.22 & 47.97 & 46.20 \\
250 & - & - & 46.15 & 36.83 \\
500 & 29.29 & 28.92 & - & - \\
750 & 00.00 & 16.00 & 31.02 & 56.70 \\
1000 & 00.00 & - & - & - \\
2000 & - & - & 80.11 & - \\
4000 & - & - & 92.41 & - \\
5000 & 55.69 & 51.19 & - & - \\
\hline
\end{tabular}

Table 2: Results on the official test sets available on CodaLabs. Numbers indicate binary-F1 for Task 5 and micro-F1 for Task 6 . indicates the model was finetuned on the specific task and indicates the model was first fine-tuned on the other task. The first line reports the results trained on the combination of the corresponding train and development sets - 7, 174 for Task 5 and 9, 452 for Task 6 . The remaining lines are based on a ensemble of the 5 models trained on the corresponding number of examples using a majority vote.

fine-tuning a Bert-based model on both tasks using a multi-task approach as opposed to the transfer learning approach employed here.

\section{Acknowledgements}

We would like to thank the anonymous reviewer for their feedback. Our experiments were conducted using computational infrastructure provided by the Barnard Vagelos Computational Science Center.

\section{References}

Jacob Devlin, Ming-Wei Chang, Kenton Lee, and Kristina Toutanova. 2019. BERT: Pre-training of deep bidirectional transformers for language understanding. In Proceedings of the 2019 Conference of the North American Chapter of the Association for Computational Linguistics: Human Language Technologies, Volume 1 (Long and Short Papers), pages 4171-4186, Minneapolis, Minnesota. Association for Computational Linguistics.

Ari Z Klein, Arjun Magge, Karen O’Connor, Jesus Ivan Flores Amaro, Davy Weissenbacher, and Graciela Gonzalez Hernandez. 2021. Toward using twitter for tracking covid-19: A natural language processing pipeline and exploratory data set. J Med Internet Res, 23(1):e25314. 
Arjun Magge, Ari Klein, Ivan Flores, Ilseyar Alimova, Mohammed Ali Al-garadi, Antonio MirandaEscalada, Zulfat Miftahutdinov, Eulàlia FarréMaduell, Salvador Lima López, Juan M Banda, Karen O'Connor, Abeed Sarker, Elena Tutubalina, Martin Krallinger, Davy Weissenbacher, and Graciela Gonzalez-Hernandez. 2021. Overview of the sixth social media mining for health applications (\# smm4h) shared tasks at naacl 2021. In Proceedings of the Sixth Social Media Mining for Health Applications Workshop \& Shared Task.

Matthew E. Peters, Sebastian Ruder, and Noah A. Smith. 2019. To tune or not to tune? adapting pretrained representations to diverse tasks. In Proceedings of the 4th Workshop on Representation Learning for NLP (RepL4NLP-2019), pages 7-14, Florence, Italy. Association for Computational Linguistics.

Sebastian Ruder. 2021. Recent Advances in Language Model Fine-tuning. http://ruder.io/ recent-advances-lm-fine-tuning.

Victor Sanh, Lysandre Debut, Julien Chaumond, and Thomas Wolf. 2019. Distilbert, a distilled version of bert: smaller, faster, cheaper and lighter. arXiv preprint arXiv:1910.01108.

Thomas Wolf, Lysandre Debut, Victor Sanh, Julien Chaumond, Clement Delangue, Anthony Moi, Pierric Cistac, Tim Rault, Remi Louf, Morgan Funtowicz, Joe Davison, Sam Shleifer, Patrick von Platen, Clara Ma, Yacine Jernite, Julien Plu, Canwen Xu, Teven Le Scao, Sylvain Gugger, Mariama Drame, Quentin Lhoest, and Alexander Rush. 2020. Transformers: State-of-the-art natural language processing. In Proceedings of the 2020 Conference on Empirical Methods in Natural Language Processing: System Demonstrations, pages 38-45, Online. Association for Computational Linguistics. 